\title{
Preserved microstructural integrity of the corticospinal tract in patients with glioma-induced motor epilepsy: a study using mean apparent propagator magnetic resonance imaging
}

\author{
Yuhui Wang ${ }^{1,2} \wedge$, Kaiji Deng ${ }^{3}$, Yifan Sun ${ }^{3}$, Xinming Huang ${ }^{3}$, Yihai Dai ${ }^{4}$, Weitao $\mathrm{Chen}^{4}$, Xiaomei $\mathrm{Hu}^{5}$, \\ Rifeng Jiang ${ }^{3 \wedge}$
}

${ }^{1}$ Department of Radiology, Union Hospital, Tongji Medical College, Huazhong University of Science and Technology, Wuhan, China; ${ }^{2}$ Hubei Province Key Laboratory of Molecular Imaging, Wuhan, China; ${ }^{3}$ Department of Radiology, Fujian Medical University Union Hospital, Fuzhou, China; ${ }^{4}$ Department of Neurosurgery, Fujian Medical University Union Hospital, Fuzhou, China; ${ }^{5}$ Department of Pathology, Fujian Medical University Union Hospital, Fuzhou, China

Contributions: (I) Conception and design: Y Wang, R Jiang; (II) Administrative support: R Jiang, X Huang; (III) Provision of study materials or patients: Y Dai, W Chen, X Hu; (IV) Collection and assembly of data: K Deng, Y Sun; (V) Data analysis and interpretation: R Jiang, X Huang; (VI) Manuscript writing: All authors; (VII) Final approval of manuscript: All authors.

Correspondence to: Rifeng Jiang. Department of Radiology, Fujian Medical University Union Hospital, 29 Xinquan Road, Fuzhou 350001, China. Email: 26630706@qq.com.

Background: To compare the microstructural integrity of the corticospinal tract (CST) between glioma patients with motor epilepsy and without epilepsy using mean apparent propagator magnetic resonance imaging (MAP-MRI).

Methods: A total of 26 patients with glioma adjacent to the CST pathway (10 with motor epilepsy and 16 without epilepsy) and 13 matched healthy controls underwent brain structural and diffusion MRI. The morphological characteristics of the CST (tract volume, tract number, and average length) were extracted, and diffusion parameter values including mean squared displacement (MSD), q-space inverse variance (QIV), return-to-origin probability (RTOP), return-to-axis probabilities (RTAP), return-to-plane probabilities (RTPP), fractional anisotropy (FA), mean diffusivity (MD), axial diffusivity (AD), and radial diffusivity (RD) along the CST were evaluated. The CST features were compared between healthy and affected sides and the relative CST features were compared across the three groups of participants. A receiver operating characteristic (ROC) curve was plotted to assess the performance of each relative CST characteristic for glioma-induced CST changes.

Results: For patients without epilepsy, the tract number, tract volume, FA, RD, MSD, QIV, and RTAP changed significantly on the affected CST side compared with those on the healthy CST side $(\mathrm{P}=0.002$, $0.002,0.0300 .017,0.039,0.044$, and 0.002 , respectively). In contrast, for patients with motor epilepsy, no significant difference was found between the affected and healthy side in almost all CST features except RTPP ( $\mathrm{P}=0.028)$. Compared with patients with motor epilepsy, the relative tract number, tract volume, $\mathrm{AD}$, and RTAP were significantly lower $(\mathrm{P}=0.027,0.018,0.040$, and 0.027 , respectively) in patients without epilepsy, and their areas under the curve (AUCs) were $0.763,0.781,0.744$, and 0.763 , respectively. No significant difference was found between patients with motor epilepsy and matched healthy controls.

Conclusions: The MAP-MRI is a promising approach for evaluating CST changes. It provides additional information reflecting the microstructural complexity of the CST and demonstrates the preserved microstructural integrity of the CST in glioma patients with motor epilepsy.

^ ORCID: Yuhui Wang, 0000-0002-2175-0711; Rifeng Jiang, 0000-0001-6959-0027. 


\begin{abstract}
Keywords: Glioma; epilepsy; corticospinal tract (CST); mean apparent propagator (MAP); diffusion tensor imaging (DTI)
\end{abstract}

Submitted Jun 28, 2021. Accepted for publication Oct 08, 2021.

doi: 10.21037/qims-21-679

View this article at: https://dx.doi.org/10.21037/qims-21-679

\section{Introduction}

Brain tumor is an important cause of epilepsy alongside other common causes, such as hippocampal sclerosis (1). Seizure presents frequently as a symptom of many types of brain tumors, especially glioma $(2,3)$. The mechanism of epileptogenesis in glioma is not fully understood, but is believed to be multifactorial (3). Electrographically, seizures arise from the peritumoral cortex in most patients with brain tumors due to changes in that specific area $(4,5)$. The role of peritumoral white matter and the tumor itself is also important. Mechanical compression of peritumoral normal tissue such as white matter leads to ischemia and hypoxia in the affected tissue, which may finally develop into an epileptic focus (6). The internal relationship of genetic factors of the tumor, such as isocitrate dehydrogenase (IDH) mutation, could also be partially responsible for seizures (7). In addition, the tumor itself secretes chemical factors that can alter the peritumoral microenvironment, which gradually leads to seizures $(3,6)$. Therefore, the peritumoral microenvironment is complex and warrants further study.

Motor seizure is a common type of tumor-associated seizure, which is characterized mainly by seizure with motor symptoms and subclassified as either simple or complex. The corticospinal tract (CST) is the principal tract for controlling primary motor activity from the motor cortex. As the CST plays an important role in the conduction of abnormal currents in patients with motor epilepsy, we hypothesized that there may be a potential association between the integrity of the CST and motor epilepsy. However, to date, no studies have investigated this association.

Diffusion tensor imaging (DTI) is a widely used method for analyzing the effects of glioma on surrounding white matter such as the CST (8-14). Apart from the morphological characteristics of the CST, DTI also provides several diffusion indices including fractional anisotropy (FA), mean diffusivity (MD), axial diffusivity (AD), and radial diffusivity (RD). However, the simplified assumption of a Gaussian spin displacement distribution in DTI weakens its ability to interpret changes of the microstructure in and around tumors due to the high heterogeneity and complexity of glioma (9).

Mean apparent propagator magnetic resonance imaging (MAP-MRI) is a recently proposed diffusion model, which connects the diffusion-weighted imaging q-space with the spin displacements (15). It can efficiently measure the probability density function (PDF) of spin displacement and quantify useful metrics of this PDF indicative of diffusion in complex microstructures (9). These metrics include mean squared displacement (MSD), q-space inverse variance (QIV), returnto-origin probability (RTOP), return-to-axis probabilities (RTAP), and return-to-plane probabilities (RTPP), which can increase the ability to differentiate tissues with distinct structural and architectural features with much less variability (16), thus better accounting for the microstructural characteristics in tissue. The RTOP, RTAP, and RTPP describe the probability of the water molecules that return to the origin, axis, and plane, respectively, with a high value denoting a constrained structure. The MSD can be treated as a high angular resolution analogue of the DTI-MD. The QIV can be interpreted as the inverse of the "variance" of q. It is equivalent to MSD for cases with Gaussian distribution; however, for non-Gaussian distribution, QIV performs similarly, which explains why the gray matter performs differently from white matter in QIV (17).

Despite the great potential of MAP-MRI in evaluating the relationship between glioma-related seizures and glioma-induced microstructural changes to the CST, until now, this approach has not been applied to this end. Therefore, the aim of this study was to employ both MAPMRI and DTI to compare the difference of CST features between glioma patients with motor epilepsy and those without epilepsy.

We present the following article in accordance with the STARD reporting checklist (available at https://dx.doi. org/10.21037/qims-21-679).

\section{Methods}

\section{Patients}

This prospective study was conducted in accordance with 


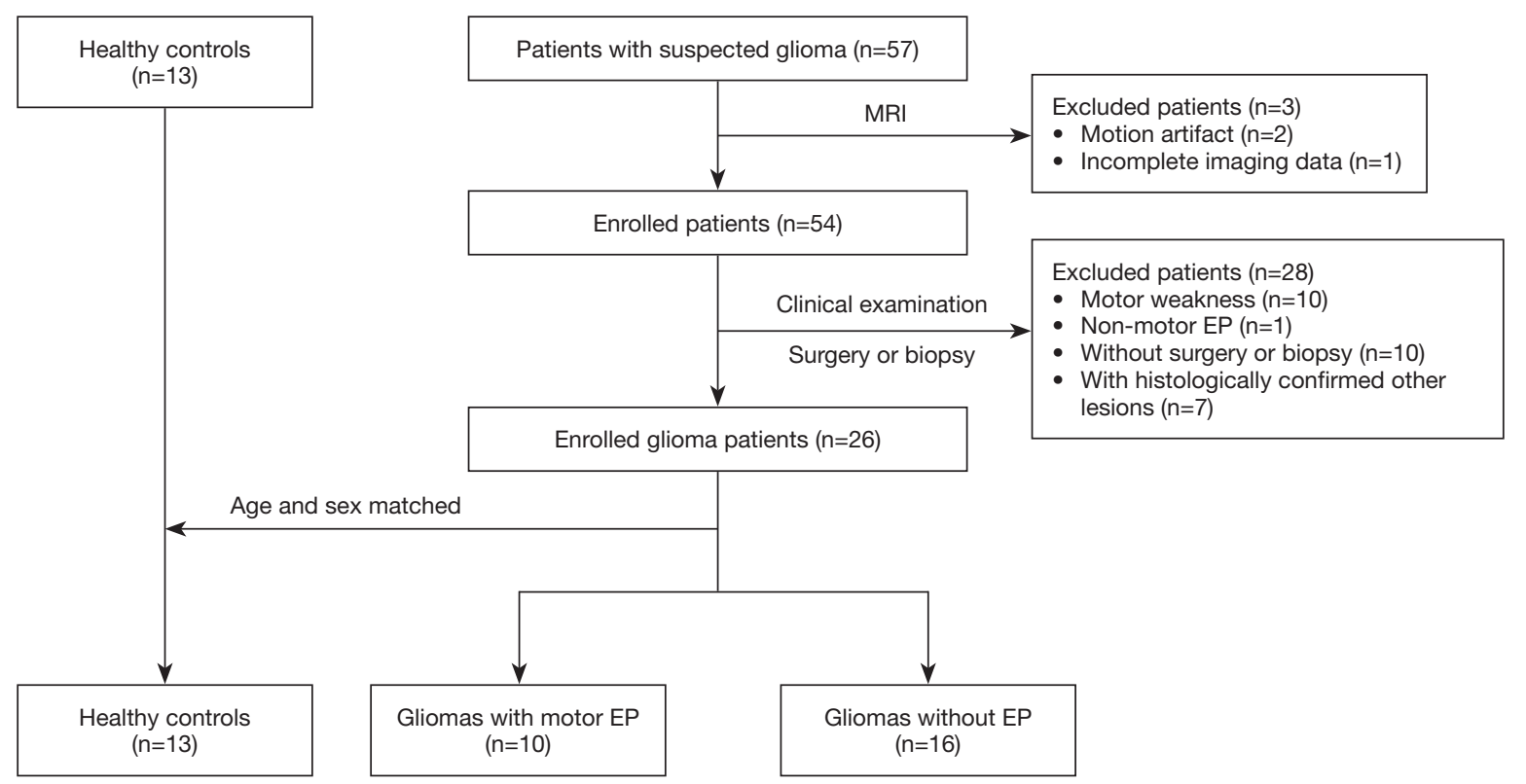

Figure 1 Screening process for the included glioma patients and healthy controls. EP, epilepsy.

the Declaration of Helsinki (as revised in 2013). The study was approved by the Ethics Committee Board of Fujian Medical University Union Hospital, and informed consent was provided by all participants.

Patients with suspected gliomas located in or adjacent to the CST pathway were enrolled in this study between March 2019 and January 2021 and underwent preoperative structural and diffusion MRI. Exclusion criteria were as follows: (I) incomplete imaging data or poor image quality such as those with motion artifacts; (II) without pathologically confirmed glioma; (III) both sides of the CST were impacted by a large tumor; (IV) non-motor epilepsy; (V) abnormal muscle strength of the upper or lower limbs; and (VI) minimal distance between the tumor and CST was more than $20 \mathrm{~mm}$.

The screening process for participants is shown in Figure 1. This study finally included 26 glioma patients, with 17 males and 9 females. The participants were then divided into two groups: motor epilepsy [10 patients, median age: 41 years, interquartile range (IQR): 32-48 years] and without epilepsy (16 patients, median age: 50.5 years, IQR: 37.5-62 years). Additionally, 13 gender- and age-matched volunteers aged 33-52 years were recruited as healthy controls.

\section{Clinical and pathological information}

Some clinical information of the participants including gender, age, duration of symptoms, and Karnofsky performance status (KPS) were acquired. The muscle strength in both upper and lower limbs was assessed using a clinical examination (grade 0-5) (18). Pathologically, the type and grade of the tumor were determined according to the World Health Organization (WHO) classification criteria (19). The IDH1R132H mutation status and Ki67 labeling index (LI) were also evaluated. Epilepsy was diagnosed according to clinical practice guidelines for the diagnosis of glioma-related epilepsy (20).

\section{MRI acquisition}

All image acquisitions were conducted on a 3T MR scanner (MAGNETOM Prisma, Siemens Healthcare, Erlangen, Germany) equipped with a 64-channel head coil. The structural MRI protocol included pre-contrast sagittal T1weighted magnetization-prepared rapid acquisition with gradient echo image (T1-MPRAGE) with a voxel size of $0.9375 \times 0.9375 \times 0.9 \mathrm{~mm}^{3}$, axial $\mathrm{T} 2$-weighted $(\mathrm{T} 2 \mathrm{~W})$ fast spin-echo (FSE) images, axial fluid-attenuated inversion recovery (FLAIR) T2W images, and subsequent contrastenhanced axial/sagittal/coronal FLAIR T1-weighted (T1W) images.

A grid sampling scheme was used to acquire 128 diffusion q-space samples, including $14 \mathrm{~b}$ values of $250,500,750$, $1,000,1,250,1,500,2,000,2,250,2,500,2,750,3,000,3,250$, 
3,500, 4,000 s/mm $\mathrm{mm}^{2}$ along 3, 6, 4, 3, 12, 12, 6, 15, 12, 12, $4,12,24$, and 3 directions, respectively. The other scan parameters were repetition time (TR), 3,900 ms; echo time (TE), $88 \mathrm{~ms}$; slice acceleration factor, 2; n GRAPPA, 2; field of view (FOV), $230 \times 230 \mathrm{~mm}^{2}$; voxel size, $2.5 \times 2.5 \times 2.5 \mathrm{~mm}^{3}$, without gap and acquisition time, $8 \mathrm{~min} 44 \mathrm{sec}$. As the eddy current artifact produced by the grid sampling scheme cannot be corrected using eddy current correction, the bipolar pulse was used to handle eddy current at the sequence level (21).

\section{Data postprocessing and evaluation}

\section{Calculation of diffusion parameter maps}

All diffusion-weighted images underwent motion correction with the Diffusion Kit eddy tool (22). Afterwards, the six independent components of the diffusion tensor were estimated, and FA, MD, AD, and RD were further calculated. The MAP-MRI parameters were calculated using software developed in-house with Python, called NeuDiLab, which is based on an open-resource tool DIPY Toolbox (https://www.dipy.org/) (23). In brief, the MAP was fit with radial order of 6 . No denoising was applied to the data prior to MAP fitting, but a positivity constraint and Laplacian regularization with a weighting of 0.05 were applied during fitting (16). These settings were recommended for robust fitting by the author of the DIPY MAP-MRI toolbox. Finally, MAP-MRI parameter maps, including MSD, QIV, RTOP, RTAP, and RTPP, were constructed.

\section{CST tracking and quantification}

The fiber pathways of the whole brain were reconstructed in DSI-studio (version 19 December 2019 build, http://dsistudio.labsolver.org/) using generalized q-sampling imaging (GQI) (24). The restricted diffusion was quantified using restricted diffusion imaging (25). The CST was tracked by two experienced radiologists (with 11 and 8 years of experience) blinded to the patient's information together with a consensus reached for each participant. The CST was determined by the selection of fibers passing through seed and target regions of interest (ROIs). The seed ROIs were placed at the anterior cerebral peduncles and the target ROIs were placed at the primary motor cortex and its underlying white matter area, with the central sulcus serving as the posterior border (26). Then, the deterministic streamline tracking algorithm, Streamline (Euler), was performed to obtain the whole CST fiber tractography using the default tracking parameters in DSI-studio. To verify the reproducibility of the measurements, 10 glioma patients were randomly selected, and the two radiologists independently repeated the tracking of the CST for them. The tract number, average tract length, and tract volume of the CST were evaluated using the "tract statistics" function in DSI-studio. Subsequently, FA, MD, AD, RD, MSD, QIV, RTOP, RTAP, and RTPP values along the CST were calculated. The relative values of the affected CST were calculated as the ratios of the FA, MD, AD, RD, MSD, QIV, RTOP, RTAP, and RTPP of the affected side to those corresponding values of the healthy side. The relative values for healthy controls were calculated as the ratios of the left side to the right side. Finally, tumor size and the minimum distance between CST and tumor were measured by an experienced radiologist based on the structural MR images and those merged with the CSTs.

\section{Evaluation of the tumor features on MRI}

The tumor size was measured by an experienced radiologist who calculated the tumor volumes slice by slice using ImageJ (https://imagej.nih.gov/ij/) on structural MR images. The total tumor size was the sum of the volumes of all tumor slices. The minimal distance between the CST and the tumor were evaluated on the structural MR images (such as T2-FLAIR) with CST marked in white, as shown in Figure S1. Moreover, cortical involvement by the tumor and abnormal peritumoral area affecting the CST were also evaluated.

\section{Statistical analysis}

The software SPSS 20.0 (IBM Corp., Armonk, NY, USA) was used for statistical analyses. Intraclass correlation coefficients were calculated to verify the reproducibility of the measurements between the two radiologists. The paired Wilcoxon test was performed to compare the differences between the CST characteristics including the tract number, average tract length, tract volume, AD, RD, FA, MD, MSD, QIV, RTAP, RTOP, and RTPP on the affected side and those on the healthy side for glioma patients, and also between the CST characteristics on the left side and those on the right side for healthy controls. The MannWhitney U test was further conducted to compare the relative CST characteristics across participants with motor epilepsy, patients without epilepsy, and healthy controls. A receiver operating characteristic (ROC) curve was finally plotted to further assess the diagnostic performance of each 
Table 1 Demographic, clinical, and pathological characteristics of the participants

\begin{tabular}{|c|c|c|c|c|}
\hline Characteristics & Glioma without EP & Glioma with motor EP & Healthy controls & $P$ value \\
\hline Age (years) & $50.50(37.50-62.00)$ & $41.00(32.00-48.00)$ & $47.00(33.00-52.00)$ & 0.253 \\
\hline Gender (male), n (\%) & $10(62.50)$ & $7(70.00)$ & $5(38.46)$ & 0.298 \\
\hline KPS & $90.00(75.00-90.00)$ & $90.00(70.00-90.00)$ & - & 0.909 \\
\hline Tumor size $\left(\mathrm{cm}^{3}\right)$ & $34.20(26.74-70.82)$ & $18.96(5.93-32.05)$ & - & $0.018^{*}$ \\
\hline Cortical involvement, n (\%) & $13(81.25)$ & $10(100.00)$ & - & 0.262 \\
\hline Abnormal peritumoral area affecting the CST, $n(\%)$ & $3(18.75)$ & $4(40.00)$ & - & 0.369 \\
\hline Minimal distance between CST and tumor (mm) & $9.42(5.38-14.28)$ & $8.81(3.36-16.41)$ & - & 0.712 \\
\hline Tumor grade, n (\%) & & & & 0.325 \\
\hline Grade II & $5(31.25)$ & $6(60.00)$ & - & \\
\hline Grade III & $3(18.75)$ & $2(20.00)$ & - & \\
\hline Grade IV & $8(50.00)$ & $2(20.00)$ & - & \\
\hline IDH1 mutation, n (\%) & $8(50.00)$ & $6(60.00)$ & - & 0.677 \\
\hline
\end{tabular}

Data were presented as median (interquartile range) or $n(\%) .{ }^{*}$, represented a statistical difference $(P<0.05)$. EP, epilepsy; KPS, Karnofsky performance status; CST, corticospinal tract; Ki-67 LI, Ki-67 labeling index; IDH, isocitrate dehydrogenase.

relative CST characteristic for glioma-induced epilepsy.

\section{Results}

\section{Demographic characteristics of participants}

A total of 39 participants were finally included in this study, including 10 glioma patients with motor epilepsy, 16 glioma patients without epilepsy, and 13 matched healthy controls. The demographic, clinical, and pathological information of participants are listed in Table 1. The time interval between MRI data collection and surgery was less than 4 weeks. No significant difference was found across the three groups for age and gender $(\mathrm{P}=0.253$ and 0.298$)$. In glioma patients, seizures were present in $54.55 \%$ (6 of 11) of participants with grade II gliomas, $40 \%$ (2 of 5) of participants with grade III gliomas, and 20\% (2 of 10) of participants with grade IV gliomas. Compared with glioma patients without epilepsy, the glioma patients with motor epilepsy showed great similarities in the KPS, disease duration, minimal distance between CST and tumor, ratio of cortical involvement, ratio of abnormal peritumoral area affecting the CST, recurrence ratio, tumor grade, and IDH1 mutation ratio ( $\mathrm{P}>0.05$ for all), but the tumor size and Ki67 LI were significantly lower in glioma patients with motor epilepsy ( $\mathrm{P}=0.018$ and 0.042$)$.

\section{Reproducibility of the CST measurements}

The intraobserver agreement was found to be good between the two radiologists for the randomly selected 10 glioma patients since the intraclass correlation coefficients were between 0.756 and 0.999 , as shown in Table S1.

\section{Changes of CST features on the affected side in glioma patients}

For all the glioma patients, the MD, RD, MSD, and QIV on the affected CST side were significantly higher compared with those on the healthy side $(\mathrm{P}=0.028,0.012$, 0.014 , and 0.012 , respectively). The tract number, tract volume, FA, and RTAP on the affected CST side were significantly lower compared with those on the healthy side 
Table 2 Changes of CST features on the affected side for all glioma patients

\begin{tabular}{|c|c|c|c|c|c|c|c|c|}
\hline CST feature & \multicolumn{4}{|c|}{ Glioma patients $(n=26)$} & \multicolumn{4}{|c|}{ Healthy controls $(n=13)$} \\
\hline Tract number & $470(196-732)$ & 794 (596-887) & -3.086 & $0.002^{*}$ & $495(483-643)$ & $629(519-863)$ & -1.782 & 0.075 \\
\hline $\begin{array}{l}\text { Mean tract length } \\
(\mathrm{cm})\end{array}$ & $\begin{array}{c}14.155 \\
(13.792-14.729)\end{array}$ & $\begin{array}{c}14.235 \\
(13.642-14.842)\end{array}$ & -0.317 & 0.751 & $\begin{array}{c}13.463 \\
(13.240-13.858)\end{array}$ & $\begin{array}{c}13.368 \\
(13.254-13.756)\end{array}$ & -0.804 & 0.422 \\
\hline Tract volume $\left(\mathrm{cm}^{3}\right)$ & 5.563 (3.749-6.568) & $7.239(6.360-8.115)$ & -3.035 & $0.002^{*}$ & $6.130(4.955-6.725)$ & $6.202(5.954-6.867)$ & -1.363 & 0.173 \\
\hline DTI-FA & $0.513(0.490-0.539)$ & $0.539(0.497-0.568)$ & -2.172 & $0.030^{*}$ & $0.515(0.500-0.535)$ & $0.522(0.503-0.542)$ & -1.153 & 0.249 \\
\hline DTI-MD & $0.500(0.483-0.518)$ & $0.491(0.484-0.502)$ & -2.197 & $0.028^{*}$ & $0.490(0.482-0.496)$ & $0.491(0.481-0.501)$ & -0.594 & 0.552 \\
\hline DTI-RD & $0.326(0.304-0.340)$ & $0.312(0.303-0.330)$ & -2.502 & $0.012^{*}$ & $0.318(0.308-0.326)$ & $0.319(0.306-0.330)$ & -0.245 & 0.807 \\
\hline MAP-RTAP & $8.500(7.420-9.137)$ & $9.119(8.327-9.496)$ & -2.908 & $0.004^{*}$ & $9.260(8.866-9.640)$ & 9.207 (8.753-9.598) & -1.153 & 0.249 \\
\hline MAP-RTOP & $4.178(3.438-6.362)$ & $4.889(4.245-6.480)$ & -0.927 & 0.354 & $8.093(6.064-10.225)$ & $8.641(5.869-14.958)$ & -0.035 & 0.972 \\
\hline MAP-RTPP & $5.250(5.099-5.327)$ & $5.268(5.152-5.367)$ & -1.257 & 0.209 & $5.390(5.321-5.409)$ & $5.373(5.353-5.410)$ & -1.013 & 0.311 \\
\hline
\end{tabular}

Data were presented as median (interquartile range). *, represented a statistical difference $(\mathrm{P}<0.05)$. CST, corticospinal tract; DTI, diffusion tensor imaging; $A D$, axial diffusivity; FA, fractional anisotropy; MD, mean diffusivity; RD, radial diffusivity; MAP, mean apparent propagator; MSD, mean squared displacement; QIV, q-space inverse variance; RTAP, return-to-axis probabilities; RTOP, return-to-origin probability; RTPP, return-to-plane probabilities.

$(\mathrm{P}=0.002,0.002,0.030$, and 0.004 , respectively). The results are shown in Table 2.

For glioma patients with motor epilepsy, no significant difference was found between the affected side and the healthy side for almost all CST features $(\mathrm{P}>0.05$ for all), with the exception of RTPP $(\mathrm{P}=0.028)$. For glioma patients without epilepsy, RD, MSD, and QIV on the affected CST side were significantly higher compared with those on the healthy side $(\mathrm{P}=0.017,0.039$, and 0.044 , respectively). The tract number, tract volume, FA, and RTAP on the affected CST side were significantly lower compared with those on the healthy side $(\mathrm{P}=0.002,0.002,0.030$, and 0.002 , respectively). The results are shown in Table 3.

No significant difference was found in all the CST features between the left and right sides for health controls $(\mathrm{P}>0.05$ for all).

\section{Changes of relative CST features in glioma patients}

There was no significant difference for all relative CST features between healthy controls and participants with motor epilepsy $(\mathrm{P}>0.05$ for all). However, compared with glioma patients with motor epilepsy, the relative CST features including the tract number, tract volume, $\mathrm{AD}$, and RTAP in glioma patients without epilepsy were significantly lower ( $\mathrm{P}=0.027,0.018,0.040$, and 0.027 , respectively). These results are shown in detail in Table 4, and the corresponding box and whisker plots are shown in Figure 2. In Figure 3 and Figure S2, 3 representative participants demonstrating the CST changes in glioma patients with motor epilepsy and without epilepsy are displayed.

\section{ROC analysis}

A ROC curve was plotted to assess the diagnostic performance of the relative CST characteristics in comparing glioma patients with motor epilepsy and those without epilepsy. In distinguishing the glioma patients with motor epilepsy and those without epilepsy, the relative tract volume had a slightly higher performance with an AUC of 0.781 (sensitivity of $70 \%$ and a specificity of $81.25 \%$ ). Tract number and MAP-RTAP came second, with AUCs of 0.763 and 0.763 , respectively (sensitivity of $80 \%$ and $80 \%$, and specificity of $75 \%$ and $81.25 \%$, respectively). This was 
Table 3 Changes of CST features on the affected side for glioma patients with motor epilepsy and patients without epilepsy

\begin{tabular}{|c|c|c|c|c|c|c|c|c|}
\hline CST feature & \multicolumn{4}{|c|}{ Glioma patients with motor epilepsy $(n=10)$} & \multicolumn{4}{|c|}{ Glioma patients without epilepsy $(n=16)$} \\
\hline Tract number & $695(437-950)$ & $801(594-872)$ & -0.561 & 0.575 & $318(86-501.5)$ & $787.5(622-911.5)$ & -3.154 & $0.002^{*}$ \\
\hline $\begin{array}{l}\text { Mean tract length } \\
(\mathrm{cm})\end{array}$ & $\begin{array}{c}13.897 \\
(13.676-14.175)\end{array}$ & $\begin{array}{c}13.587 \\
(13.325-14.079)\end{array}$ & -0.764 & 0.445 & $\begin{array}{c}14.430 \\
(14.025-15.011)\end{array}$ & $\begin{array}{c}14.558 \\
(14.235-15.122)\end{array}$ & -0.827 & 0.408 \\
\hline Tract volume $\left(\mathrm{cm}^{3}\right)$ & $6.224(5.347-7.305)$ & $6.847(5.583-7.878)$ & -0.357 & 0.721 & $4.833(2.455-6.057)$ & $7.239(6.561-8.271)$ & -3.103 & $0.002^{*}$ \\
\hline DTI-FA & $0.504(0.462-0.534)$ & $0.496(0.471-0.568)$ & -.051 & 0.959 & $0.516(0.509-0.540)$ & $0.542(0.521-0.563)$ & -2.172 & $0.030^{\star}$ \\
\hline DTI-MD & $0.506(0.48-0.519)$ & $0.494(0.483-0.502)$ & -1.376 & 0.169 & $0.500(0.490-0.510)$ & $0.491(0.486-0.503)$ & -1.706 & 0.088 \\
\hline DTI-RD & $0.326(0.305-0.349)$ & $0.316(0.303-0.338)$ & -0.866 & 0.386 & $0.326(0.304-0.336)$ & $0.310(0.300-0.315)$ & -2.379 & $0.017^{\star}$ \\
\hline MAP-RTAP & 8.703 (7.388-9.357) & 9.049 (7.522-9.307) & -0.255 & 0.799 & 8.381 (7.656-9.073) & $9.134(8.361-9.524)$ & -3.051 & $0.002^{*}$ \\
\hline MAP-RTOP & $4.058(3.438-4.981)$ & $4.261(3.931-5.427)$ & -1.580 & 0.114 & $4.718(3.432-12.630)$ & $5.485(4.473-7.947)$ & -0.052 & 0.959 \\
\hline MAP-RTPP & $5.247(5.127-5.294)$ & $5.334(5.152-5.376)$ & -2.191 & $0.028^{*}$ & $5.250(5.067-5.354)$ & $5.240(5.139-5.352)$ & -0.362 & 0.717 \\
\hline
\end{tabular}

Data were presented as median (interquartile range). *, represented a statistical difference $(\mathrm{P}<0.05)$. CST, corticospinal tract; DTI, diffusion tensor imaging; AD, axial diffusivity; FA, fractional anisotropy; MD, mean diffusivity; RD, radial diffusivity; MAP, mean apparent propagator; MSD, mean squared displacement; QIV, q-space inverse variance; RTAP, return-to-axis probabilities; RTOP, return-to-origin probability; RTPP, return-to-plane probabilities.

Table 4 Changes of relative CST features in glioma patients with motor epilepsy compared with those without epilepsy

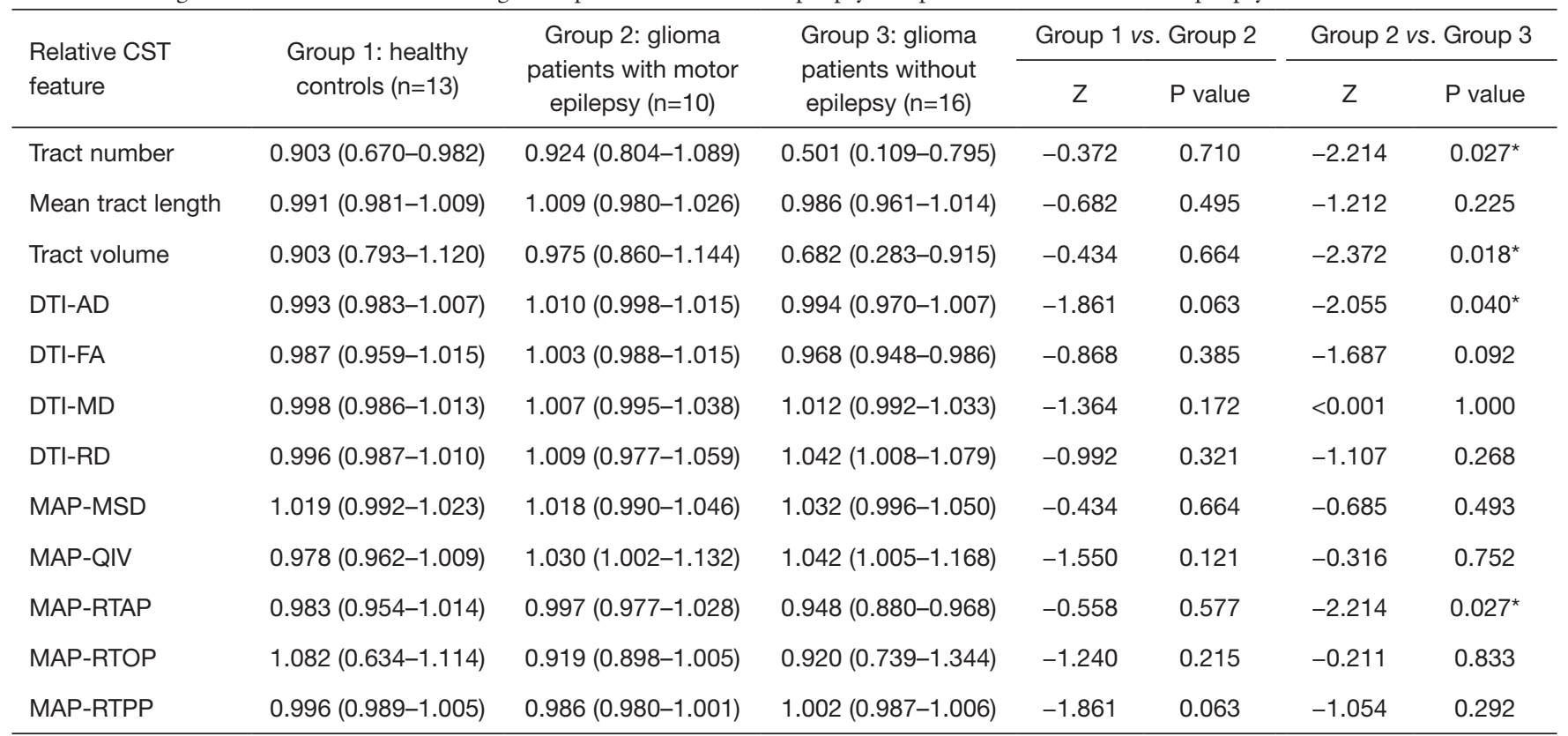

Data were presented as median (interquartile range). *, represented a statistical difference $(\mathrm{P}<0.05)$. CST, corticospinal tract; DTI, diffusion tensor imaging; AD, axial diffusivity; FA, fractional anisotropy; MD, mean diffusivity; RD, radial diffusivity; MAP, mean apparent propagator; MSD, mean squared displacement; QIV, q-space inverse variance; RTAP, return-to-axis probabilities; RTOP, return-to-origin probability; RTPP, return-to-plane probabilities. 

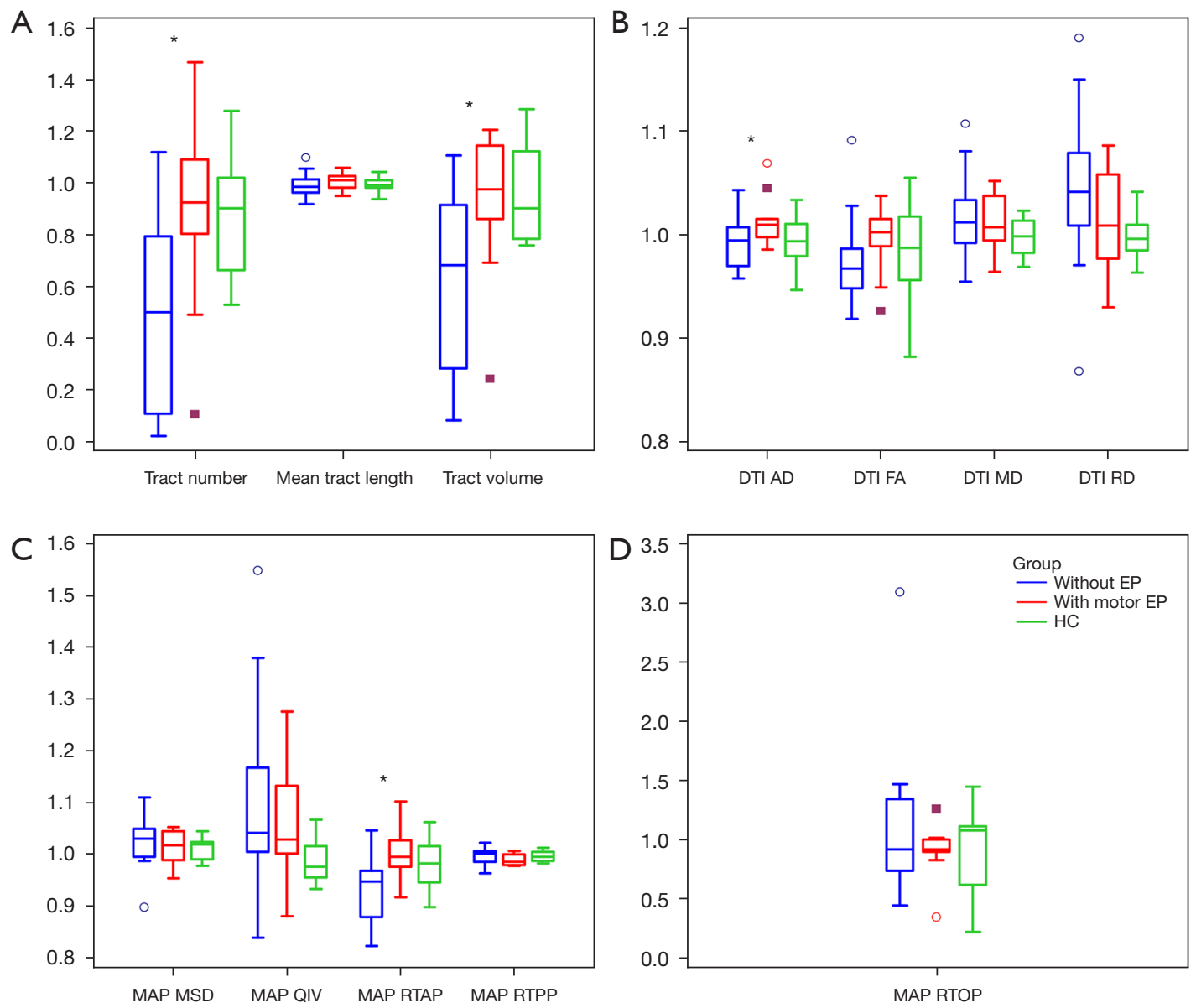

Figure 2 Changes of relative CST features in glioma patients and healthy controls. Box and whisker plots of the relative morphological (A), DTI (B), and MAP-MRI (C,D) features of the CST which showed that the relative CST features including the tract number, tract volume, DTI-AD, and MAP-RTAP in glioma patients without epilepsy were significantly lower compared with glioma patients with motor epilepsy $\left.{ }^{*}\right)$. In contrast, no significant changes were found in all the relative CST features between healthy controls and glioma patients with motor epilepsy. CST, corticospinal tract; DTI, diffusion tensor imaging; MAP, mean apparent propagator; MRI, magnetic resonance imaging; $\mathrm{AD}$, axial diffusivity; RTAP, return-to-axis probabilities; FA, fractional anisotropy; MD, mean diffusivity; RD, radial diffusivity; MSD, mean squared displacement; QIV, q-space inverse variance; RTPP, return-to-plane probabilities; RTOP, return-to-origin probability; EP, epilepsy; $\mathrm{HC}$, healthy control.

followed by DTI-AD, with an AUC of 0.744 (a sensitivity of $70 \%$ and a specificity of $75 \%$ ), as shown in Figure 4 . The other relative features had relatively low AUCs, as shown in Table 5 .

\section{Discussion}

In this study, both MAP-MRI and DTI were employed to compare the CST features between the affected and healthy side, and compare the relative CST features across healthy controls, glioma patients with motor epilepsy, and those without epilepsy. It was found that many CST features changed significantly on the affected side in glioma patients, especially in those without epilepsy. Moreover, some relative CST features also changed significantly in patients without epilepsy.

For glioma patients without epilepsy, RD, MSD, and QIV were found to be significantly higher on the affected CST side, whereas the tract number, tract volume, FA, and RTAP were significantly lower on the affected CST side compared with those on the healthy side. Moreover, the relative CST features including the tract number, tract volume, $\mathrm{AD}$, and 

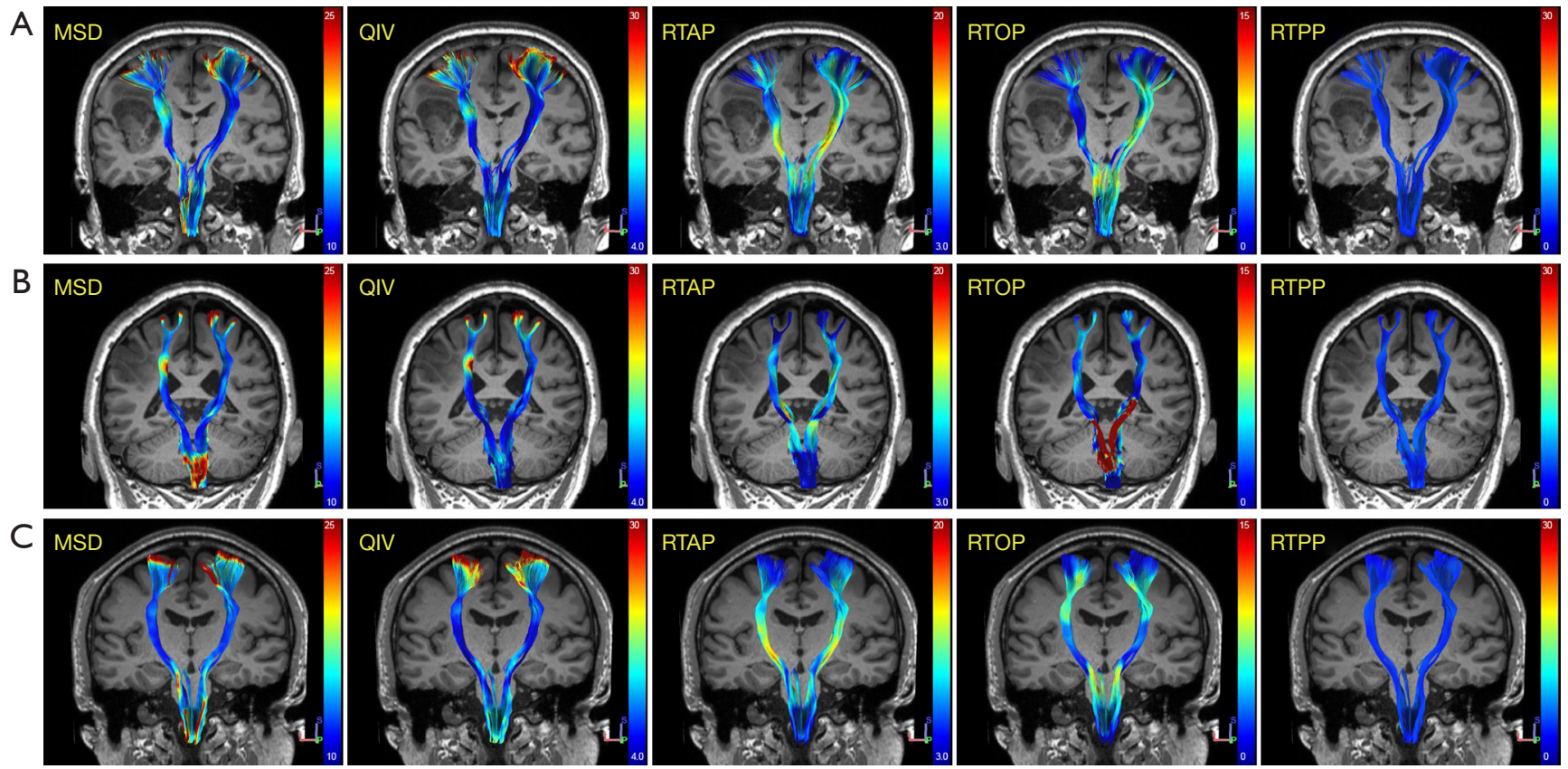

Figure 3 The MAP-MRI features of CST marked on pre-contrast 3D T1W images demonstrating the changes of the CST in glioma patients with motor epilepsy and without epilepsy in 3 representative participants. The patient (A) was a 57-year-old male with oligodendroglioma (WHO grade II), but without epilepsy. On the affected CST side, diffuse and obvious changes were found in MSD (increase), QIV (increase), RTAP (decrease), and RTOP (decrease), whereas change was not obvious in RTPP. The patient (B) was a 70-year-old male with diffuse astrocytoma (WHO grade II) and motor epilepsy. On the affected CST side, a focal increase was found in MSD and QIV, but changes were not obvious in RTAP, RTOP, and RTPP. The subject (C) was a 54-year-old healthy male. All the CST features had good bilateral symmetry. MAP, mean apparent propagator; MRI, magnetic resonance imaging; CST, corticospinal tract; T1W, T1-weighted; WHO, World Health Organization; MSD, mean squared displacement; QIV, q-space inverse variance; RTAP, return-to-axis probabilities; RTOP, return-to-origin probability; RTPP, return-to-plane probabilities.

RTAP were significantly higher in glioma patients without epilepsy. These changes suggest that the integrity of the CST might have been damaged in glioma patients without epilepsy, but the changes were not significant enough to cause a decrease in muscle strength, as all participants in this study had normal muscle strength. Gliomas vary in nuclear pleomorphism, hypercellularity, endothelial proliferation, and microvascular density, which may result in the disruption of myelin and axonal membranes in and around the tumor location and explain the corresponding imaging changes on the affected CST side.

For glioma patients with motor epilepsy, no significant difference was found between the affected side and the healthy side for almost all CST features except the RTPP, and no significant difference was found for all the relative CST features compared with healthy controls. These changes may indicate that the integrity of the CST is well preserved in glioma patients with motor epilepsy, which may be one reason for the finding that glioma patients with motor epilepsy had better prognoses in previous studies. It was reported that the occurrence of epileptic seizures independently impacted glioma prognosis, as both progression-free survival and overall survival were longer in glioma patients with a history of epileptic seizures for both low-grade glioma and high-grade glioma (6,27-29).

The relative CST features (AD and RTAP) were significantly higher in patients without epilepsy compared with those in patients with motor epilepsy, indicating that axial diffusion changes can serve as markers of gliomarelated invasive change. The ROC curve demonstrated that RTAP had an AUC similar to that of AD, indicating that MAP-MRI parameters have a similar sensitivity to the DTI parameter for detecting integrity of the CST. However, for glioma patients with motor epilepsy, only RTPP decreased significantly on the affected side compared with the healthy side, which may indicate that MAP-MRI parameters may 

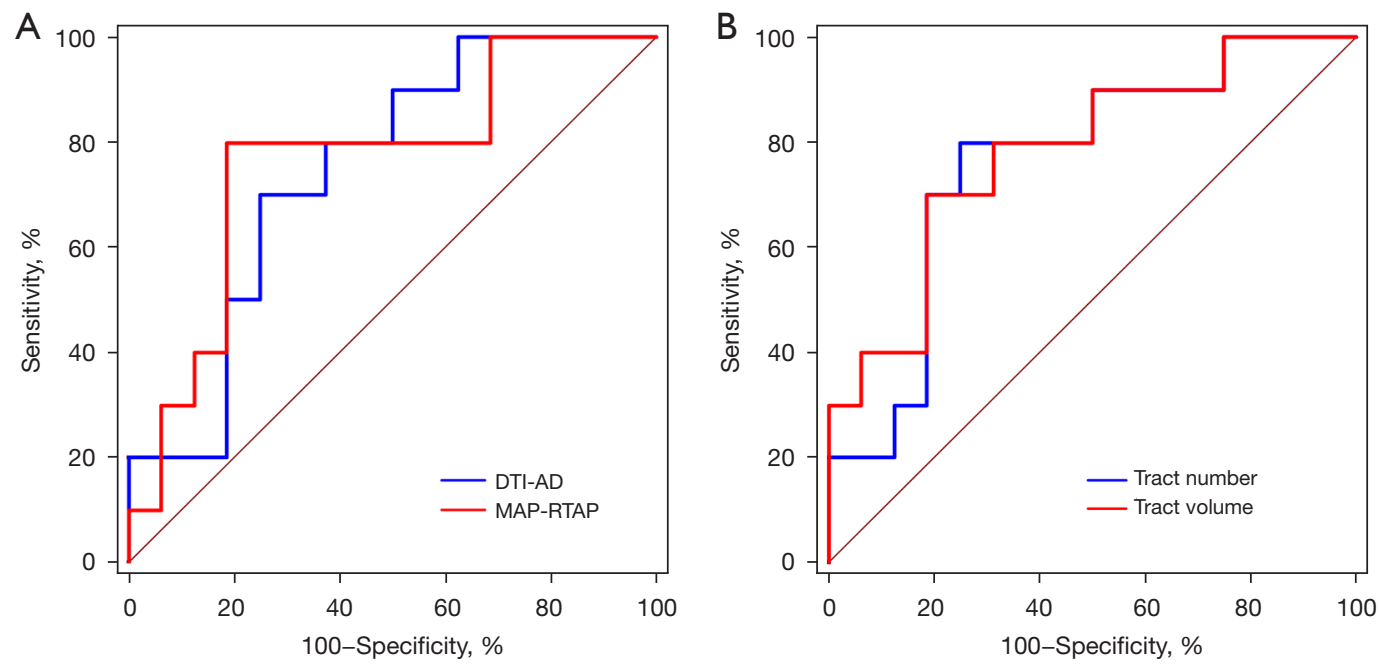

Figure 4 ROC curves for the differentiation of glioma patients with motor epilepsy from those without epilepsy. ROC curves distinguishing the glioma patients with motor epilepsy and without epilepsy showed that relative DTI-AD and MAP-RTAP had AUCs of 0.744 and 0.763, respectively (A). The tract number and tract volume also had similar AUCs of 0.763 and 0.781 , respectively (B). ROC, receiver operating characteristic curve; DTI, diffusion tensor imaging; AD, axial diffusivity; MAP, mean apparent propagator; RTAP, return-to-axis probabilities; AUC, area under the curve.

Table 5 ROC diagnostic performance of the relative CST features in evaluating the CST changes between patients with motor epilepsy and those without epilepsy

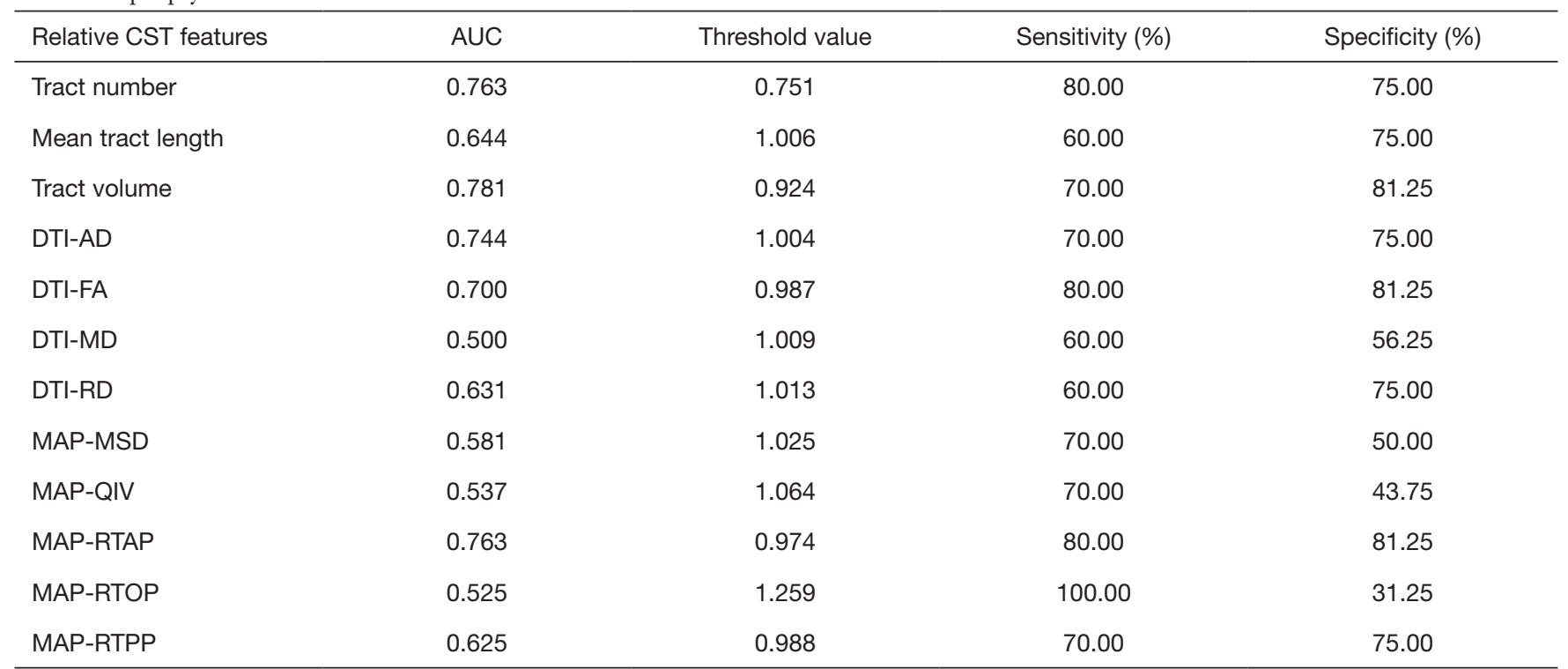

ROC, receiver operating characteristic curve; CST, corticospinal tract; AUC, area under the curve; DTI, diffusion tensor imaging; AD, axial diffusivity; FA, fractional anisotropy; MD, mean diffusivity; RD, radial diffusivity; MAP, mean apparent propagator; MSD, mean squared displacement; QIV, q-space inverse variance; RTAP, return-to-axis probabilities; RTOP, return-to-origin probability; RTPP, return-to-plane probabilities. 
be more sensitive in demonstrating the subtle changes in the CST microenvironment for glioma patients with motor epilepsy. These MAP parameters that encode directional information were shown to be suitable for characterizing complex diffusion in anisotropic tissues and had great potential in distinguishing white matter biomarkers for axonal loss or demyelination (16).

Some confounding factors were excluded in this study. Firstly, glioma patients with motor weakness were excluded, as damage of the CST caused by glioma can result in motor weakness $(8,12)$ and some features of the CST were significantly different between patients with motor weakness and those of the normal group $(12,26)$. In addition, it has been reported that CST features such as FA vary across individuals and ages but have good bilateral symmetry (30). Therefore, the relative CST features were taken to minimize the effects of age and individual variation. Furthermore, the minimal distance from tumor to CST, cortical involvement of the tumor, and abnormal peritumoral area affecting the CST may constitute other probable factors. However, in this study, no significant difference was found in the minimal distance between tumor and CST, ratio of cortical involvement, and abnormal peritumoral area affecting the CST between glioma patients with motor epilepsy and those without epilepsy, as shown in Table 1.

In addition, this study revealed that seizures were present in $54.55 \%$ ( 6 of 11$)$ of participants with grade II gliomas, $40 \%$ ( 2 of 5 ) of those with grade III gliomas, and 20\% ( 2 of 10) of those with grade IV gliomas, which is similar to the findings of previous studies $(3,31)$. As an indicator of cellular proliferation, the Ki-67 LI was found to be significantly lower in patients with motor epilepsy compared with that in those without epilepsy. This finding was also consistent with the previously reported values (6). Smaller tumor size was found in participants with motor epilepsy. We speculate that smaller or slow-growing tumors provide enough time for seizures to develop, whereas fast-growing tumors easily destroy the integrity of the CST, thus affecting the conduction of epileptic currents. This was consistent with a previous study of high-grade tumors (32). However, the reverse was found with low-grade tumors in other studies, where patients with large tumors were more likely to present with seizures than those with small tumors (33). Due to our small sample size, a larger cohort is of great necessity to draw further conclusions.

There are some limitations to this study. Tumor grade, motor weakness, and cortical involvement by the tumor were confounding factors in this study. We followed strict inclusion and exclusion criteria to obtain homogenous data and excluded some of the possible confounding factors such as motor weakness. However, subgroup analyses for other factors were not possible due to the small sample size involved, especially for the participants with motor epilepsy. Therefore, a larger cohort may be needed to draw a further conclusion. Another limitation is that there were 7 participants with abnormal peritumoral areas affecting the CST. An abnormal peritumoral area with T2-hyperintensity can be edema or a non-contrast enhancing tumor, and the diffusion parameters of the CST might be affected if the abnormal peritumoral area is masked with the CST. However, Fisher's exact test did not reveal a significant difference in the ratio of abnormal peritumoral area affecting the CST between glioma patients with motor epilepsy and those without epilepsy. Therefore, we believe the conclusions are still credible and valuable for clinical practice.

\section{Conclusions}

The MAP-MRI is a promising method to evaluate CST changes in glioma patients by providing additional information on microstructural complexity. It can also demonstrate the preserved microstructural integrity of the CST in glioma patients with motor epilepsy, which may further elucidate the mechanism of motor epilepsy associated with glioma.

\section{Acknowledgments}

Funding: This work was supported by grants from the National Natural Science Foundation of China (81701655), Joint Funds for the Innovation of Science and Technology, Fujian Province (grant number: 2017Y9024), and University Project Funded by the Education Department of Fujian Province (JK2017019).

\section{Footnote}

Reporting Checklist: The authors have completed the STARD reporting checklist. Available at https://dx.doi. org/10.21037/qims-21-679

Conflicts of Interest: All authors have completed the ICMJE uniform disclosure form (available at https://dx.doi. org/10.21037/qims-21-679). The authors have no conflicts of interest to declare. 
Ethical Statement: The authors are accountable for all aspects of the work in ensuring that questions related to the accuracy or integrity of any part of the work are appropriately investigated and resolved. The study was conducted in accordance with the Declaration of Helsinki (as revised in 2013). The study was approved by the ethics committee board of Fujian Medical University Union Hospital, and informed consent was provided by all participants.

Open Access Statement: This is an Open Access article distributed in accordance with the Creative Commons Attribution-NonCommercial-NoDerivs 4.0 International License (CC BY-NC-ND 4.0), which permits the noncommercial replication and distribution of the article with the strict proviso that no changes or edits are made and the original work is properly cited (including links to both the formal publication through the relevant DOI and the license). See: https://creativecommons.org/licenses/by-nc-nd/4.0/.

\section{References}

1. Mo J, Zhao B, Adler S, Zhang J, Shao X, Ma Y, Sang L, Hu W, Zhang C, Wang Y, Wang X, Liu C, Zhang $\mathrm{K}$. Quantitative assessment of structural and functional changes in temporal lobe epilepsy with hippocampal sclerosis. Quant Imaging Med Surg 2021;11:1782-95.

2. Rudà R, Trevisan E, Soffietti R. Epilepsy and brain tumors. Curr Opin Oncol 2010;22:611-20.

3. Armstrong TS, Grant R, Gilbert MR, Lee JW, Norden AD. Epilepsy in glioma patients: mechanisms, management, and impact of anticonvulsant therapy. Neuro Oncol 2016;18:779-89.

4. Köhling R, Senner V, Paulus W, Speckmann EJ. Epileptiform activity preferentially arises outside tumor invasion zone in glioma xenotransplants. Neurobiol Dis 2006;22:64-75.

5. de Groot M, Reijneveld JC, Aronica E, Heimans JJ. Epilepsy in patients with a brain tumour: focal epilepsy requires focused treatment. Brain 2012;135:1002-16.

6. Yang P, You G, Zhang W, Wang Y, Yao K, Jiang T. Correlation of preoperative seizures with clinicopathological factors and prognosis in anaplastic gliomas: a report of 198 patients from China. Seizure 2014;23:844-51.

7. Yang Y, Mao Q, Wang X, Liu Y, Mao Y, Zhou Q, Luo J. An analysis of 170 glioma patients and systematic review to investigate the association between IDH-1 mutations and preoperative glioma-related epilepsy. J Clin Neurosci 2016;31:56-62.

8. Gao B, Shen X, Shiroishi MS, Pang M, Li Z, Yu B, Shen G. A pilot study of pre-operative motor dysfunction from gliomas in the region of corticospinal tract: Evaluation with diffusion tensor imaging. PLoS One 2017;12:e0182795.

9. Avram AV, Sarlls JE, Barnett AS, Ozarslan E, Thomas C, Irfanoglu MO, Hutchinson E, Pierpaoli C, Basser PJ. Clinical feasibility of using mean apparent propagator (MAP) MRI to characterize brain tissue microstructure. Neuroimage 2016;127:422-34.

10. Jia X, Su Z, Hu J, Xia H, Ma H, Wang X, Yan J, Ma D. The value of diffusion tensor tractography delineating corticospinal tract in glioma in rat: validation via correlation histology. PeerJ 2019;7:e6453.

11. Henderson F, Abdullah KG, Verma R, Brem S. Tractography and the connectome in neurosurgical treatment of gliomas: the premise, the progress, and the potential. Neurosurg Focus 2020;48:E6.

12. Min ZG, Niu C, Zhang QL, Zhang M, Qian YC. Optimal factors of diffusion tensor imaging predicting corticospinal tract injury in patients with brain tumors. Korean J Radiol 2017;18:844-51.

13. Cepeda S, Garcia-Garcia S, Arrese I, Velasco-Casares $\mathrm{M}$, Sarabia R. Acute changes in diffusion tensor-derived metrics and its correlation with the motor outcome in gliomas adjacent to the corticospinal tract. Surg Neurol Int 2021;12:51.

14. Laundre BJ, Jellison BJ, Badie B, Alexander AL, Field AS. Diffusion tensor imaging of the corticospinal tract before and after mass resection as correlated with clinical motor findings: preliminary data. AJNR Am J Neuroradiol 2005;26:791-6.

15. Özarslan E, Koay CG, Shepherd TM, Komlosh ME, Irfanoglu MO, Pierpaoli C, Basser PJ. Mean apparent propagator (MAP) MRI: a novel diffusion imaging method for mapping tissue microstructure. Neuroimage 2013;78:16-32.

16. Fick RHJ, Wassermann D, Caruyer E, Deriche R. MAPL: Tissue microstructure estimation using Laplacianregularized MAP-MRI and its application to HCP data. Neuroimage 2016;134:365-85.

17. Wu YC, Field AS, Alexander AL. Computation of diffusion function measures in q-space using magnetic resonance hybrid diffusion imaging. IEEE Trans Med Imaging 2008;27:858-65.

18. Cuthbert SC, Goodheart GJ, Jr. On the reliability and 
validity of manual muscle testing: a literature review. Chiropr Osteopat 2007;15:4.

19. Louis DN, Perry A, Reifenberger G, von Deimling A, Figarella-Branger D, Cavenee WK, Ohgaki H, Wiestler OD, Kleihues P, Ellison DW. The 2016 World Health Organization Classification of Tumors of the Central Nervous System: a summary. Acta Neuropathol 2016;131:803-20.

20. Liang S, Fan X, Zhao M, Shan X, Li W, Ding P, You G, Hong Z, Yang X, Luan G, Ma W, Yang H, You Y, Yang T, Li L, Liao W, Wang L, Wu X, Yu X, Zhang J, et al. Clinical practice guidelines for the diagnosis and treatment of adult diffuse glioma-related epilepsy. Cancer Med 2019;8:4527-35.

21. Miao HC, Wu MT, Kao EF, Chiu YH, Chou MC. Comparisons of reproducibility and mean values of diffusion tensor imaging-derived indices between unipolar and bipolar diffusion pulse sequences. J Neuroimaging 2015;25:892-9.

22. Xie S, Chen L, Zuo N, Jiang T. DiffusionKit: A light onestop solution for diffusion MRI data analysis. J Neurosci Methods 2016;273:107-19.

23. Garyfallidis E, Brett M, Amirbekian B, Rokem A, van der Walt S, Descoteaux M, Nimmo-Smith I. Dipy, a library for the analysis of diffusion MRI data. Front Neuroinform 2014;8:8.

24. Melhem ER, Mori S, Mukundan G, Kraut MA, Pomper MG, van Zijl PC. Diffusion tensor MR imaging of the brain and white matter tractography. AJR Am J Roentgenol 2002;178:3-16.

25. Yeh FC, Liu L, Hitchens TK, Wu YL. Mapping immune cell infiltration using restricted diffusion MRI. Magn Reson Med 2017;77:603-12.

26. Jiang R, Jiang S, Song S, Wei X, Deng K, Zhang Z, Xue Y. Laplacian-regularized mean apparent propagator-MRI in evaluating corticospinal tract injury in patients with brain glioma. Korean J Radiol 2021;22:759-69.

27. Pallud J, Audureau E, Blonski M, Sanai N, Bauchet L, Fontaine D, Mandonnet E, Dezamis E, Psimaras D, Guyotat J, Peruzzi P, Page P, Gal B, Parraga E, Baron MH, Vlaicu M, Guillevin R, Devaux B, Duffau H, Taillandier L, et al. Epileptic seizures in diffuse low-grade gliomas in adults. Brain 2014;137:449-62.

28. Yu Z, Zhang N, Hameed NUF, Qiu T, Zhuang D, Lu J, $\mathrm{Wu} \mathrm{J}$. The analysis of risk factors and survival outcome for Chinese patients with epilepsy with high-grade glioma. World Neurosurg 2019;125:e947-57.

29. Pignatti F, van den Bent M, Curran D, Debruyne C, Sylvester R, Therasse P, Afra D, Cornu P, Bolla M, Vecht C, Karim AB; European Organization for Research and Treatment of Cancer Brain Tumor Cooperative Group; European Organization for Research and Treatment of Cancer Radiotherapy Cooperative Group. Prognostic factors for survival in adult patients with cerebral lowgrade glioma. J Clin Oncol 2002;20:2076-84.

30. Reich DS, Smith SA, Jones CK, Zackowski KM, van Zijl PC, Calabresi PA, Mori S. Quantitative characterization of the corticospinal tract at $3 \mathrm{~T}$. AJNR Am J Neuroradiol 2006;27:2168-78.

31. Zhou XW, Wang X, Yang Y, Luo JW, Dong H, Liu YH, Mao Q. Biomarkers related with seizure risk in glioma patients: a systematic review. Clin Neurol Neurosurg 2016;151:113-9.

32. Lee JW, Wen PY, Hurwitz S, Black P, Kesari S, Drappatz J, Golby AJ, Wells WM, 3rd, Warfield SK, Kikinis R, Bromfield EB. Morphological characteristics of brain tumors causing seizures. Arch Neurol 2010;67:336-42.

33. Rudà R, Bello L, Duffau H, Soffietti R. Seizures in lowgrade gliomas: natural history, pathogenesis, and outcome after treatments. Neuro Oncol 2012;14 Suppl 4:iv55-64.
Cite this article as: Wang Y, Deng K, Sun Y, Huang X, Dai Y, Chen W, Hu X, Jiang R. Preserved microstructural integrity of the corticospinal tract in patients with glioma-induced motor epilepsy: a study using mean apparent propagator magnetic resonance imaging. Quant Imaging Med Surg 2022;12(2):14151427. doi: 10.21037/qims-21-679 


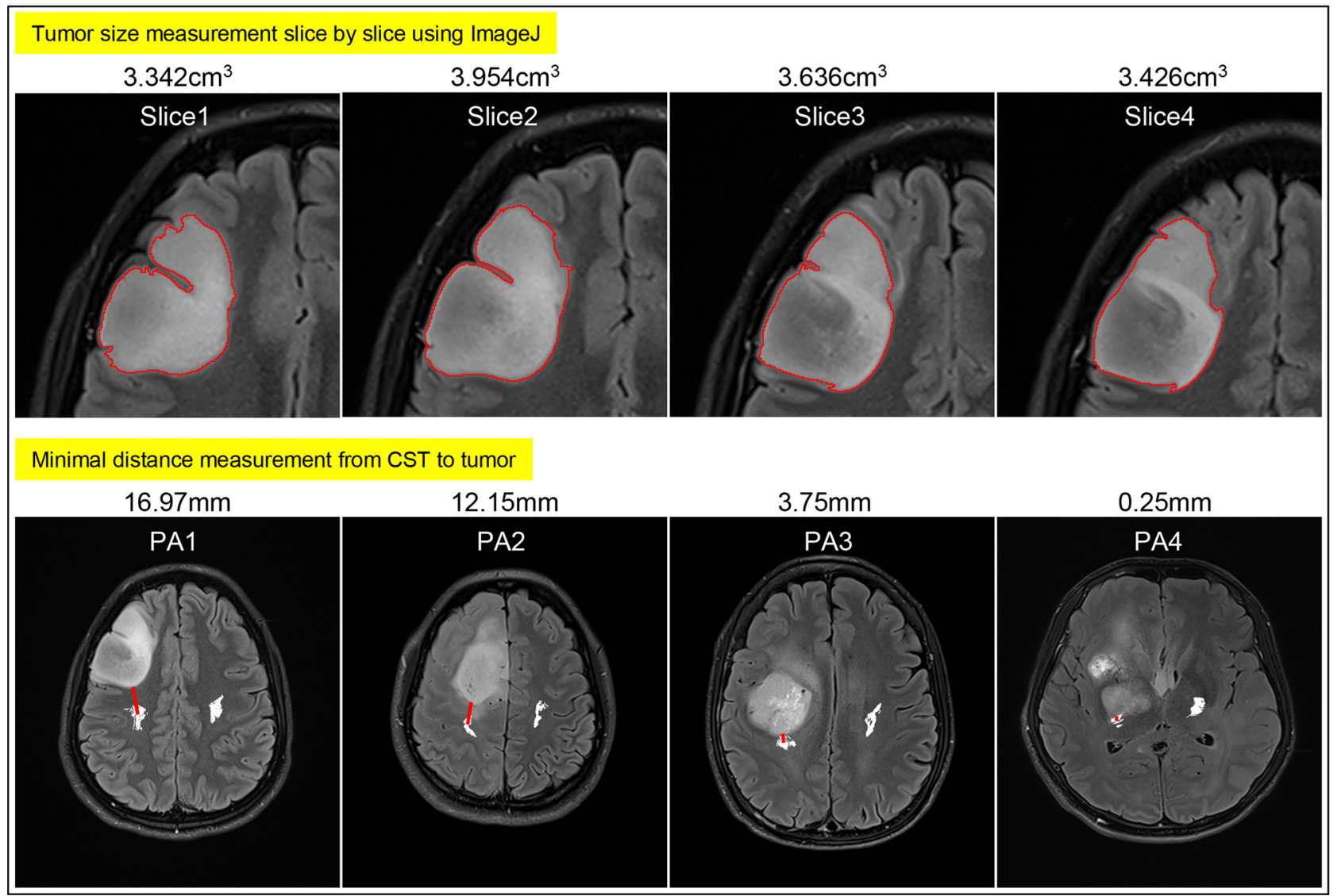

Figure S1 Measuring the tumor size and the minimal distance between the CST and the tumor. Tumor size was measured by an experienced radiologist who calculated the size slice by slice using ImageJ on structural MR images such as T2-FLAIR as shown in the upper row. The total tumor size was the sum of the volumes of all tumor slices. The minimal distance between the CST and the tumor was evaluated on structural MR images (such as T2-FLAIR) with CST marked in white, as shown in the bottom row. CST, corticospinal tract; MR, magnetic resonance; FLAIR, fluid-attenuated inversion recovery. 

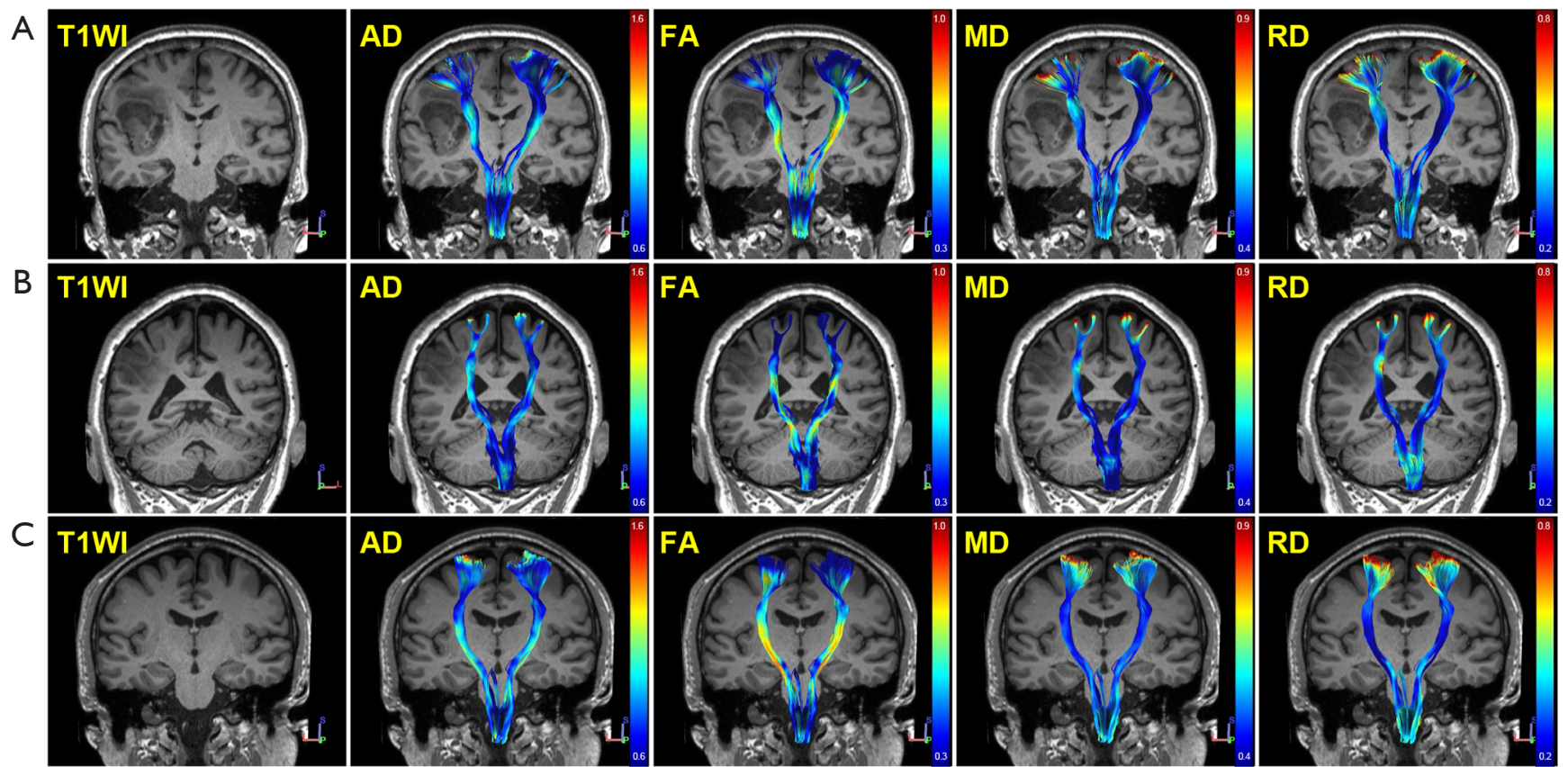

Figure S2 Changes of the CST in glioma patients with motor epilepsy and without epilepsy demonstrated by the DTI features of the CST marked on pre-contrast 3D T1W images of 3 representative participants. The patient (A) was a 57-year-old male with oligodendroglioma (WHO grade II), but without epilepsy. On the affected CST side, a diffuse and obvious increase was found in AD, MD, and RD, whereas change was not obvious in FA. The patient (B) was a 70-year-old male with diffuse astrocytoma (WHO grade II) and motor epilepsy. On the affected CST side, a focal increase was found in MD and RD, but no obvious changes were found in AD and FA. The participant (C) was a 54-year-old healthy male. All the CST features had good bilateral symmetry. CST, corticospinal tract; DTI, diffusion tensor imaging; T1W, T1-weighted; WHO, World Health Organization; AD, axial diffusivity; MD, mean diffusivity; RD, radial diffusivity; FA, fractional anisotropy. 
Table S1 Inter-observer variability of measurements for 10 randomly selected glioma patients

\begin{tabular}{|c|c|c|c|}
\hline Region & CST feature & Intraclass correlation coefficient $(95 \% \mathrm{Cl})$ & $P$ value \\
\hline \multirow{7}{*}{ Left CST } & Mean tract length $(\mathrm{cm})$ & $0.993(0.971-0.998)$ & $<0.001$ \\
\hline & Tract volume $\left(\mathrm{cm}^{3}\right)$ & $0.756(0.283-0.933)$ & 0.004 \\
\hline & DTI-AD $\left(\times 10^{3} \mathrm{~mm}^{-2} / \mathrm{s}\right)$ & $0.994(0.976-0.999)$ & $<0.001$ \\
\hline & DTI-MD $\left(\times 10^{3} \mathrm{~mm}^{-2} / \mathrm{s}\right)$ & $0.998(0.993-1.000)$ & $<0.001$ \\
\hline & DTI-RD $\left(\times 10^{3} \mathrm{~mm}^{-2} / \mathrm{s}\right)$ & $0.995(0.979-0.999)$ & $<0.001$ \\
\hline & MAP-MSD $\left(\times 10^{-5} \mathrm{~mm}^{2} / \mathrm{s}\right)$ & $0.991(0.964-0.998)$ & $<0.001$ \\
\hline & MAP-QIV $\left(\times 10^{-10} \mathrm{~mm}^{5} / \mathrm{s}\right)$ & $0.996(0.984-0.999)$ & $<0.001$ \\
\hline \multirow[t]{7}{*}{ Right CST } & Tract number & $0.904(0.663-0.975)$ & $<0.001$ \\
\hline & Mean tract length $(\mathrm{cm})$ & $0.997(0.988-0.999)$ & $<0.001$ \\
\hline & Tract volume $\left(\mathrm{cm}^{3}\right)$ & $0.860(0.535-0.963)$ & $<0.001$ \\
\hline & DTI-AD $\left(\times 10^{3} \mathrm{~mm}^{-2} / \mathrm{s}\right)$ & $0.991(0.966-0.998)$ & $<0.001$ \\
\hline & DTI-FA & $0.998(0.990-0.999)$ & $<0.001$ \\
\hline & 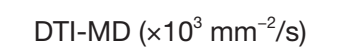 & $0.997(0.988-0.999)$ & $<0.001$ \\
\hline & DTI-RD $\left(\times 10^{3} \mathrm{~mm}^{-2} / \mathrm{s}\right)$ & $0.995(0.981-0.999)$ & $<0.001$ \\
\hline
\end{tabular}

$\mathrm{Cl}$, confidence interval; CST, corticospinal tract; DTI, diffusion tensor imaging; AD, axial diffusivity; FA, fractional anisotropy; MD, mean diffusivity; RD, radial diffusivity; MAP, mean apparent propagator; MSD, mean squared displacement; QIV, q-space inverse variance; RTAP, return-to-axis probabilities; RTOP, return-to-origin probability; RTPP, return-to-plane probabilities. 